\title{
Spectroscopic Ellipsometry and White-Light Interferometry Investigation into Time-Dependent Oxidation Rates of Uranium in Pure Oxygen
}

\author{
Yaakov Idell $^{1 *}$, Wigbert Siekhaus ${ }^{1}$ and William McLean II $^{1}$ \\ 1. Lawrence Livermore National Laboratory, Materials Science Division, Livermore, CA, USA. \\ * Corresponding author: idell1@1lnl.gov
}

Material degradation of uranium resulting from the uranium-oxygen reaction has long been of great interest to the nuclear industry in hopes of solving long-term storage and disposal related concerns. Uranium reacts with oxygen as follows: $U+\left(\frac{2+x}{2}\right) O_{2} \rightarrow U O_{2+x}$. At temperatures up to $200{ }^{\circ} \mathrm{C}$, the oxide formed is a hyperstoichiometric uranium dioxide $\left(\mathrm{UO}_{2}\right)$, with $\mathrm{x}$ in the range of 0.2 to 0.4 [1]; while at higher temperatures $\left(>275^{\circ} \mathrm{C}\right), \mathrm{U}_{3} \mathrm{O}_{8}$ is formed in addition to the $\mathrm{UO}_{2}$. Initially, there is a thin protective oxide film present $(<1 \mu \mathrm{m})$. The rate of oxidation for a thin oxide film follows a parabolic time-dependent growth rate that exhibits a growth rate behavior that is inversely proportional to the oxide thickness and is controlled by the diffusion of oxygen ions through the oxide [2]. During this period of oxide growth, elastic strain is gradually formed due to the oxide-metal density mismatch. At a certain oxide layer thickness, the strain reaches a critical level, and the oxide layer begins to crack. Upon the onset of cracking, the oxide growth is accelerated towards a linear or para-linear time dependence, which has been reported to occur at approximately $1 \mu \mathrm{m}$ [3]. The oxidation kinetics for thick oxide films $(>1 \mu \mathrm{m})$ is determined by both oxygen diffusion through a thin adherent oxide layer and the reaction of the outer surface of this oxide, which forms a porous unprotective outer layer [4]. It is not well understood precisely when this transition from a thin oxide to thick oxide occurs. Additionally, most of the previous studies regarding oxide growth in a uranium-oxygen system has been conducted with dry air [5]. Previous researchers have proposed because nitrogen does not react with uranium at low temperatures $\left(<300^{\circ} \mathrm{C}\right)$; therefore, the uranium-dry air reaction is essentially the same as the uranium-oxygen reaction.

We investigated the oxide layer kinetics through in-situ spectroscopic ellipsometry (SE) and white-light interferometry (WLI) to determine the thickness at which the time-dependent growth rates transition from parabolic to linear. Additionally, we report and discuss differences in the oxidation kinetics of uranium in a pure oxygen environment against the literature of dry air. The sample was inserted into an environmental cell that was capable of being heated up to $300^{\circ} \mathrm{C}$ while flowing $99.95 \%$ oxygen gas. Several experiments were conducted at different temperatures ranging from $60{ }^{\circ} \mathrm{C}$ to $185^{\circ} \mathrm{C}$ in order to capture the transition thickness of the time-dependent growth rates from parabolic to linear. Prior to running in-situ SE measurements, it was necessary to experimentally determine the optical constants for uranium and $\mathrm{UO}_{2}$, which were determined to have excellent agreement with previously published values [6]. Figure 1 shows our experimentally determined oxidation growth rates where we observed only a parabolic growth rate. The activation energy of $\mathrm{UO}_{2}$ in pure $\mathrm{O}_{2}$ was determined to be $51.19 \pm 4.25 \frac{\mathrm{kJ}}{\mathrm{mol}}$ with a rate equation of $\ln k=9.637-\frac{6157}{T}$. The associated oxide growth function in pure $\mathrm{O}_{2}$ was determined to be $T=$ $10.185+e^{\frac{51187.2}{8.314}\left(\frac{1}{107+2}-\frac{1}{T+273}\right)}(2.9229 \sqrt{x})$.

The initial data suggests that the parabolic-only oxidation growth rates might be associated with the characterization technique used. Previous studies have typically used x-ray diffraction or weight gain measurements to describe the oxide growth mechanics. These measurements are volume fraction 
techniques that determine the amount of $\mathrm{UO}_{2}$ on the uranium substrate, which will account for any spalled material and newly formed oxide within small localized cracks in addition to the bulk oxide film; whereas, SE and WLI measure only the bulk oxide film thickness. The oxide layer is known to crack due to epitaxial stresses permitting $\mathrm{O}_{2}$ to rapidly bypass the protective oxide film and interact with the uranium substrate through the locally cracked areas. Previous studies have suggested that the transition from a parabolic to linear oxidation growth rate occurs during the onset of oxide cracking [5]. However, our initial results indicate no change from a parabolic to linear oxide growth rate at any point. This suggests that the previously reported linear oxide growth rate is mostly likely a pseudo-linear growth rate resulting from the superposition of multiple parabolic growth rates. It is hypothesized that the observed pseudo-linear growth is a result of the combination of the slow bulk oxide film parabolic growth rate and the faster localized parabolic growth rate where localized cracking and spalling has occurred leading to newly exposed regions of the uranium substrate [7].

\section{References:}

[1] S Orman in "Phys. Metall. Uranium Alloy.", p. 815.

[2] JG Schnizlein et al., J. Electrochem. Soc. Absorbed Electrochem. Technol. 107 (1960).

[3] GW McGillivray, DA Geeson and RC Greenwood, J. Nucl. Mater. 208 (1994), p. 81.

[4] EW Haycock, J. Electrochem. Soc. 106 (1959), p. 771.

[5] AG Ritchie, J. Nucl. Mater. 102 (1981), p. 170.

[6] S Lin et al., Surf. Interface Anal. An Int. J. Devoted to Dev. Appl. Tech. Anal. Surfaces, Interfaces Thin Film. 40 (2008), p. 645.

[7] This work was performed under the auspices of the U.S. Department of Energy by Lawrence Livermore National Laboratory under Contract DE-AC52-07NA27344.

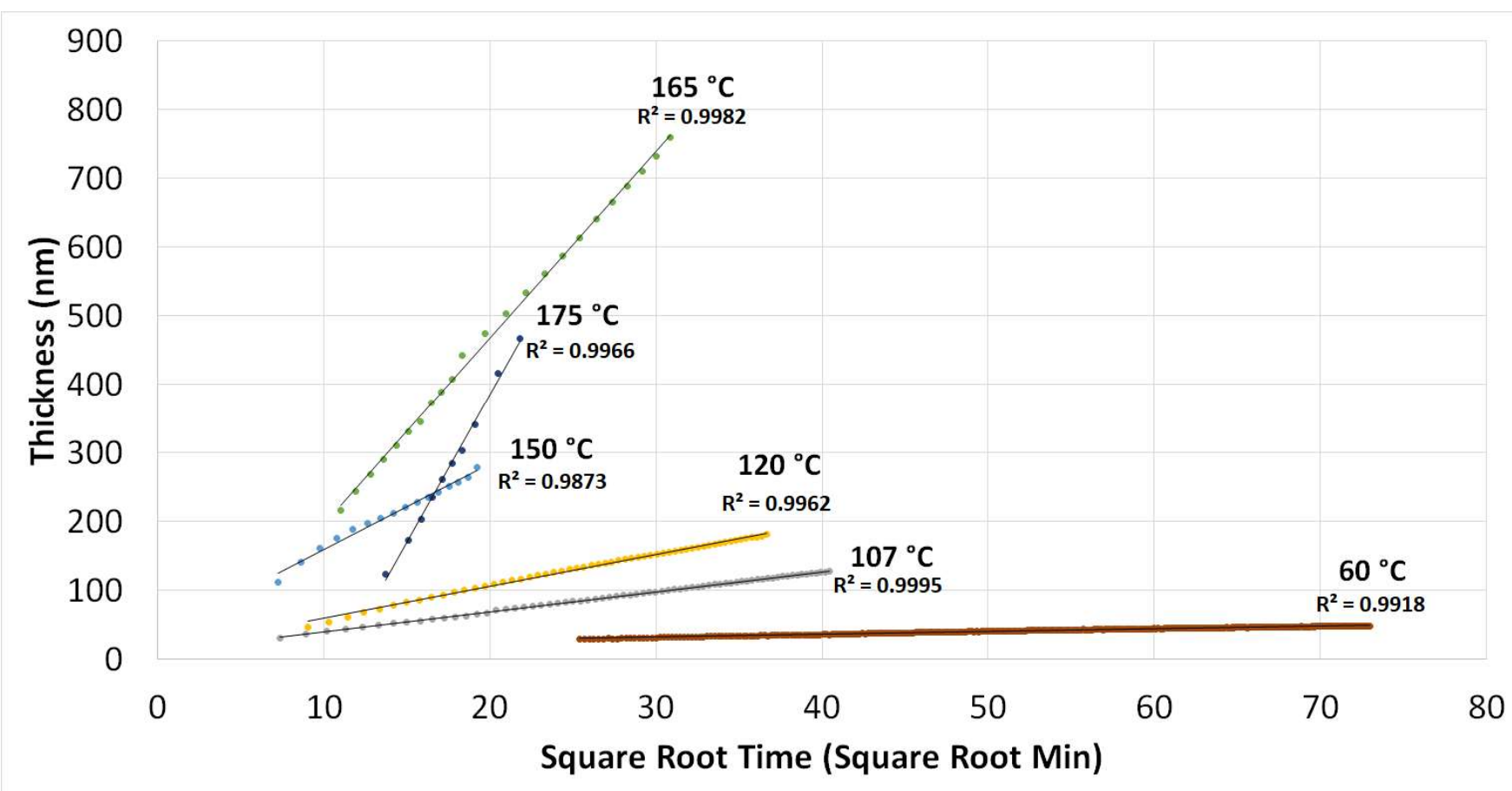

Figure 1. Oxidization growth as determined by spectroscopy ellipsometry in $99.95 \% \mathrm{O}_{2}$ at different temperatures ranging from $60{ }^{\circ} \mathrm{C}$ to $185^{\circ} \mathrm{C}$ in order to capture the transition thickness of the timedependent growth rates from parabolic to linear; however, only a parabolic growth rate was observed. 